\title{
Chernoff Bound and Saddlepoint Approximation for Outage Probability in IRS-Assisted Wireless Systems
}

\author{
Tianxiong Wang*, Gaojie Chen ${ }^{\dagger}$, Justin P. Coon*, and Mihai-Alin Badiu* \\ *Department of Engineering Science, University of Oxford, Oxford OX1 3PJ, United Kingdom \\ ${ }^{5} 5$ GIC \& 6GIC, Institute for Communication Systems (ICS), University of Surrey, Guildford GU2 7XH, United Kingdom \\ e-mail: \{tianxiong.wang, justin.coon, mihai.badiu\}@eng.ox.ac.uk, gaojie.chen@surrey.ac.uk
}

\begin{abstract}
We analyze the outage probability of an intelligent reflecting surface (IRS)-assisted communication network. An upper bound on the outage probability is formulated based on the Chernoff inequality. Furthermore, through an exact asymptotic (a large number of reflecting elements) analysis based on a saddlepoint approximation, we derive closed-form expressions of the outage probability for systems with and without a direct link and obtain the corresponding diversity orders. Simulation results corroborate our theoretical analysis and show the inaccuracies inherent in using the central limit theorem (CLT) to analyze system performance. Our analysis is accurate even for a small number of IRS elements in the high signal-to-noise ratio (SNR) regime.
\end{abstract}

Index Terms-Intelligent reflecting surface, outage probability, Chernoff bound, saddlepoint approximation

\section{INTRODUCTION}

Recently, intelligent reflecting surfaces (IRS) have been considered as an emerging technology for the physical layer of next-generation wireless communication systems [1]. An IRS comprises a large number of low-cost reflecting elements, which can be used to change the phases of incident waves for different functionalities, such as adding the reflected waves constructively for an intended user and destructively for other users [2]. Compared with other related technologies, such as relaying, IRS can facilitate energy-efficient communication since, ideally, there is no power consumed by the reflecting elements [3]. Owing to its prominent benefits, IRS-assisted communications are able to support key applications in 5G and beyond communication systems, such as Vehicle-toEverything (V2X) communications [4] and Internet of Things (IoT) [5].

An IRS-assisted communication system can significantly improve communication performance and enhance security [6]. Therefore, it is crucial to study the performance of IRS technology accurately. To date, many research efforts have been paid to the performance analysis of IRS-assisted networks from the perspectives of ergodic capacity, channel distribution and outage probability. For example, [7] and [8] analyzed the upper bound of the ergodic spectral efficiency. The capacity degradation caused by phase adjustment errors was investigated and quantified in [9]. As an essential metric to evaluate the reliability of the system, the outage probability has been studied in several works. The authors in [10] derived an upper bound of the outage probability of an IRSassisted system without a direct link based on the central limit theorem (CLT). As a step further, the work in [11] took into consideration the phase errors and showed that the channel distribution is equivalent to Nakagami fading. However, the CLT is only accurate for a large number of reflecting elements and can lead to significant approximation errors in the high SNR regime [12]. To avoid these CLT issues, the authors in [13], [14] used a gamma distribution to approximate the fading of each reflecting path. The gamma-based framework appears to offer a more accurate result. However, the gammabased approach provides neither a bound nor an asymptotic result for the outage probability. In [15], [16], the integralform outage probability was expressed by using the Gil-Pelaez inversion formula [17]. However, closed form expressions were not obtained and the infinite integration domain of the Gil-Pelaez integral makes the numerical integration difficult.

This paper investigates the outage probability of an IRSassisted system with/without a direct link. First, we use Chernoff's generic bounding technique to derive an upper bound on the outage probability, where the optimal parameter of the Chernoff bound is obtained using the gradient descent method (GDM). The obtained bound is tighter compared to the CLT based methods in high SNR regime, as showed by the simulation results. Then, to further investigate the outage probability for a large number of reflecting elements and high SNR, we derive an asymptotically accurate, closed-form expression by using the saddlepoint approximation. While in this paper we focus on double-Rayleigh distributed channel coefficients, the aforementioned methods can also be used for other channel distributions, such as double-Nakagami-m and double-Rician.

\section{SYSTEM MODEL}

We focus on an IRS with $N$ reflecting elements, labelled as $\mathrm{R}_{n}, n \in\{1,2, \ldots, N\}$, which assists the communication between a source node (S) and a destination node (D). S and $\mathrm{D}$ are equipped with one antenna and operate in the halfduplex mode. The IRS is assumed to work in the far-field of both $\mathrm{S}$ and $\mathrm{D}$, therefore, the distances between $\mathrm{S}$ to the $R_{n}$ are equal for all $n$ and the distances between the $R_{n}$ to $\mathrm{D}$ are also the same for any $n$. 
Let $h_{1 n}, h_{2 n}$ and $h_{L}$ denote the channel coefficients for the $\mathrm{S}$-to- $\mathrm{R}_{n}$, the $\mathrm{R}_{n}$-to-D and the $\mathrm{S}$-to-D channels, respectively, which are independent, circularly symmetric, complex normal random variables, each with zero mean and unit variance. Hence, the magnitudes of $h_{1 n}, h_{2 n}$ and $h_{L}$ follow the Rayleigh distribution, same as in [5], [13], [14], [18], [19], with scale parameter $\sigma=\frac{1}{\sqrt{2}}$. The independent Rayleigh fading assumption is valid if a half-wavelength-spaced linear IRS is set in an isotropic scattering environment [20]. Thus, the signal received at $\mathrm{D}$ can be given by

$$
y=\sqrt{P}\left(\sum_{n=1}^{N} d_{1}^{-v_{1} / 2} h_{1 n} e^{j \theta_{n}} d_{2}^{-v_{2} / 2} h_{2 n}+d_{L}^{-v_{L} / 2} h_{L}\right) x+u,
$$

where $x$ is the transmitted symbol with zero mean and unit power and $P$ represents the transmit power of $\mathrm{S}$. $u \sim \mathcal{C N}\left(0, \sigma_{u}^{2}\right)$ denotes the additive Gaussian white noise (AWGN) received by D. $\theta_{n}, n \in\{1,2, \ldots, N\}$ are the phases of the reflecting elements. $d_{L}, d_{1}$ and $d_{2}$ are the distances between $\mathrm{S}$ to $\mathrm{D}, \mathrm{S}$ to the IRS, and the IRS to D, respectively. $v_{L}, v_{1}$ and $v_{2}$ are the corresponding path loss coefficients. Each reflecting element is assumed to have unit reflection coefficient.

\section{A. Perfect Phase Alignment without the Direct Link}

If the direct link is blocked by obstacles, such as trees and buildings, which is more likely to happen when the system operates at high frequencies, the received SNR can reach its $\operatorname{maximum}^{1}$ when $\theta_{n}=-\arg \left(h_{1 n}\right)-\arg \left(h_{2 n}\right)$, and (1) can be rewritten as

$$
y_{1}=H_{1} \sqrt{P} d_{1}^{-v_{1} / 2} d_{2}^{-v_{2} / 2} x+u,
$$

where $H_{1}=\sum_{n=1}^{N}\left|h_{1 n}\right|\left|h_{2 n}\right|$. Denoting $\beta_{R}=d_{1}^{-v_{1}} d_{2}^{-v_{2}}$, the received SNR is

$$
\gamma_{1}=\frac{P}{\sigma_{u}^{2}} H_{1}^{2} \beta_{R}=H_{1}^{2} \gamma_{t} \beta_{R},
$$

where $\gamma_{t}=P / \sigma_{u}^{2}$ is the transmit SNR.

\section{B. Perfect Phase Alignment with the Direct Link}

When the direct link is present, the phase of the $n$th reflecting path should be aligned to the phase of the direct link, i.e., $\theta_{n}=\arg \left(h_{L}\right)-\arg \left(h_{1 n}\right)-\arg \left(h_{2 n}\right)$. Thus, the received signal can be expressed as

$$
y_{2}=H_{2} e^{j \arg \left(h_{L}\right)} \sqrt{P \beta_{R}} x+u,
$$

where $\mathrm{H}_{2}$ denotes the composite channel, which is give by $H_{2}=\sum_{n=1}^{N}\left|h_{1 n}\right|\left|h_{2 n}\right|+\sqrt{\alpha}_{L}\left|h_{L}\right|$, where $\alpha_{L}=d_{L}^{-v_{L}} / \beta_{R}$. Then, the corresponding received SNR is given by

$$
\gamma_{2}=H_{2}^{2} \gamma_{t} \beta_{R} .
$$

\footnotetext{
${ }^{1}$ In this paper, we apply the continuous phase shifts design the same as in [10] to show a clear benchmark for the outage probability in high SNR region. The practical discrete phase shift will be investigated in our future work.
}

\section{OUtage Probability ANAlysis}

The outage probability is an important measure used to evaluate the reliability of the system, which is defined as

$$
P_{\text {out }}^{(i)}(\bar{\gamma})=\mathbb{P}\left(\gamma_{i}<\bar{\gamma}\right)=F_{\gamma_{i}}(\bar{\gamma}),
$$

where $F_{\gamma_{i}}(\cdot)$ stands for the $\mathrm{CDF}$ of $\gamma_{i} ; \bar{\gamma}$ denotes the threshold SNR; $i \in\{1,2\}$ corresponds to the scenarios without and with the direct link, respectively. The outage probability for a certain threshold $\bar{\gamma}$ is equivalent to the probability that the channel coefficient falls below a value related to $\bar{\gamma}$, i.e.,

$$
P_{\text {out }}(\bar{\gamma})=F_{H_{1}}\left(\sqrt{\frac{\bar{\gamma}}{\gamma_{t} \beta_{R}}}\right) .
$$

Based on the outage probability, we can write the diversity order of the system as

$$
d_{i}=\lim _{\gamma_{t} \rightarrow \infty}-\frac{\log P_{\text {out }}^{(i)}}{\log \gamma_{t}} .
$$

Therefore, in what follows, we will focus on the CDF of the channel coefficients.

\section{A. Perfect Phase Alignment without the Direct Link}

1) Chernoff Bound: Let $G_{n}=\left|h_{1 n}\right|\left|h_{2 n}\right|$. Since $\left|h_{1 n}\right|$ and $\left|h_{2 n}\right|$ follow the independent Rayleigh distribution with the parameter $\sigma=\frac{1}{\sqrt{2}}$, the probability density function (PDF) of $G_{n}$ is [21]

$$
f_{G_{n}}(x)=4 x K_{0}(2 x),
$$

where $K_{0}(\cdot)$ denotes the modified bessel function of the second kind with order zero. A Chernoff upper bound on the CDF of $H_{1}$, denoted as $F_{H_{1}}(s)$, can be written as

$$
F_{H_{1}}(s) \leq \min _{t>0} e^{t s} \prod_{n=1}^{N} \mathrm{E}\left[e^{-t G_{n}}\right],
$$

where $\mathrm{E}\left[e^{-t G_{n}}\right]$ can be calculated to be

$$
\mathrm{E}\left[e^{-t G_{n}}\right]=\left\{\begin{array}{cl}
\frac{1}{1-\frac{t^{2}}{4}}-\frac{t \arccos \left(\frac{t}{2}\right)}{2\left(1-\frac{t^{2}}{4}\right)^{3 / 2}}, & t>0 \& t \neq 2 \\
\frac{1}{3}, & t=2 .
\end{array}\right.
$$

Denoting $w(t)=e^{t s} \prod_{n=1}^{N} \mathrm{E}\left[e^{-t G_{n}}\right]$, we are now in the position to find the optimal value of $t$ which can minimize $w(t)$. Fortunately, $w(t)$ is proved to be convex in $t$.

Lemma 1. $w(t)$ is convex in $t$.

Proof: Since $G_{n}, n=1,2, \ldots, N$ are independent random variables, $w(t)$ can be rewritten as

$$
\begin{aligned}
w(t) & =e^{t s} \mathrm{E}\left[e^{-t \sum_{n=1}^{N} G_{n}}\right] \\
& =\int_{0}^{+\infty} f_{H_{1}}(x) e^{t(s-x)} \mathrm{d} x,
\end{aligned}
$$

where $f_{H_{1}}(x)$ denotes the probability density function (PDF) of $H_{1}$. Due to the fact that $e^{t(s-x)}$ is convex in $t$ and $f_{H_{1}}(x) \geq$ $0, w(t)$ is convex in $t[22$, sec. 3.2]. 


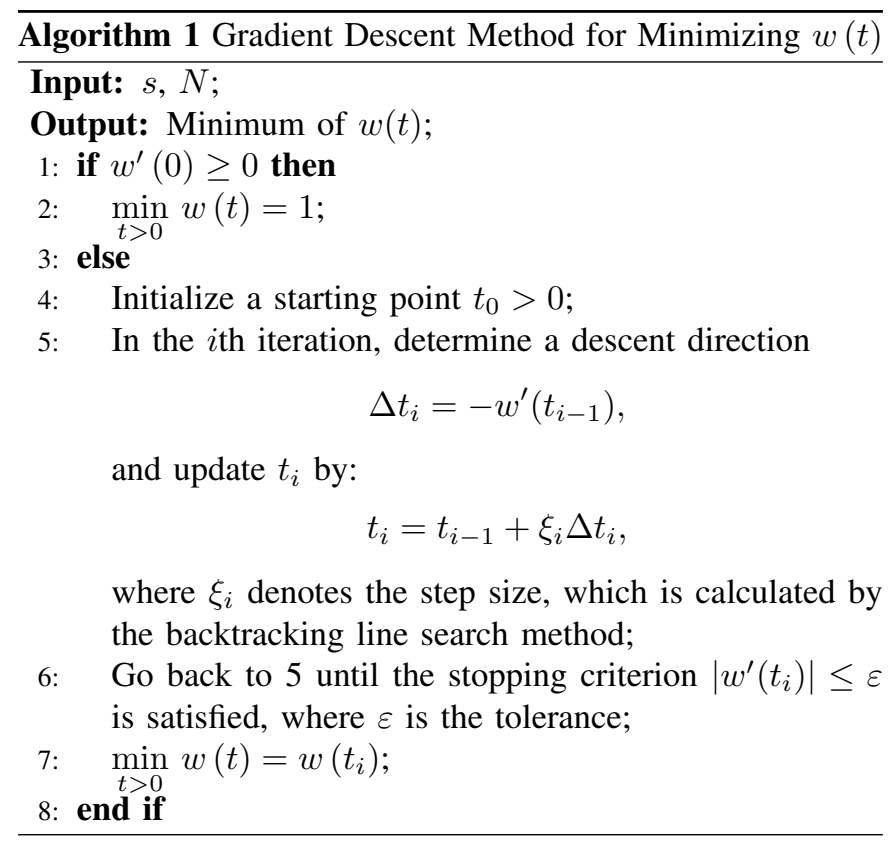

Remark 1. It should be noted that $w(t)$ is convex as long as $G_{n}, n=1,2, \ldots, N$ are independent. In other words, the Chernoff bound can be applied to other distribution of $G_{n}$, e.g., double Rice distribution.

It is mathematically intractable to obtain a close-form expression of the minimum of $w(t)$. Alternatively, we propose a gradient descent algorithm to find an approximated numerical result, as shown in Algorithm 1.

2) Saddlepoint Approximation: We again treat the case of perfect phase adjustment without a direct link. As defined before, $G_{n}=\left|h_{1 n}\right|\left|h_{2 n}\right|$. We require the distribution of $H_{1}=$ $\sum_{n=1}^{N} G_{n}$ for large $N$. Moreover, our interest lies in the tails of the distribution where the central limit approximation is not accurate. Hence, we resort to a saddlepoint approximation. The result is summarised in the following proposition.

Proposition 1. The cumulative distribution function of $H_{1}$ obeys the asymptotic equivalence

$$
\begin{aligned}
F_{H_{1}}(s) \sim & \frac{2^{N} N !}{N^{N} \sqrt{4 \pi N}} e^{-2 N\left(\ln \left(\frac{2 N}{s}\right)-1\right)} \\
& \times \sum_{n=0}^{N} \frac{\left(2 N\left(\ln \left(\frac{2 N}{s}\right)-1\right)\right)^{n}}{n !}, \quad N \rightarrow \infty .
\end{aligned}
$$

Proof: We give a proof based on the saddlepoint approximation. Let $L_{H_{1}}(t)=\mathrm{E}\left[e^{-t H_{1}}\right]$ represent the Laplace transform of the PDF of $H_{1}$, denoted as $f_{H_{1}}(x)$. This is well defined due to the fact that $G_{n} \geq 0$ for $n=1, \ldots, N$ and that fact that the Laplace transform of the PDF corresponding to the random variable $G_{n}$, denoted by $L_{G}(t)$, exists. Indeed, we can write $L_{H_{1}}(t)=L_{G}(t)^{N}$ due to independence.

Now, the probability density function of $H_{1}$ can be written as

$$
f_{H_{1}}(x)=\frac{1}{2 \pi i} \int_{c-i \infty}^{c+i \infty} e^{N \lambda(t)} \mathrm{d} t
$$

where $c$ is greater than the real part of the singularities of the integrand and

$$
\lambda(t)=\ln L_{G}(t)+\frac{x}{N} t .
$$

The integral is dominated for large $N$ by the saddlepoint of $\lambda(t)$. Denote this saddlepoint of $\lambda(t)$ by $\hat{t}$. We have that

$$
Q(\hat{t}):=-\frac{L_{G}^{\prime}(\hat{t})}{L_{G}(\hat{t})}=\frac{x}{N}
$$

must hold. For large $N$, we must have that $\hat{t}$ is large, since $L_{G}^{\prime}(\hat{t}) \approx 0$. Hence, we expand $Q(t)$ for a large argument with positive real part. It is straightforward to show that

$$
Q(t) \sim \frac{2}{t}, \quad \Re\{t\} \rightarrow \infty .
$$

It follows that

$$
\hat{t} \sim \frac{2 N}{x}, \quad N \rightarrow \infty .
$$

By considering the second derivative of $\lambda(t)$ evaluated at large $t$, it is possible to show that, indeed, a maximum occurs at $\hat{t}$.

Deforming the integral contour to pass $\hat{t}$ and using the approximation $\lambda(t) \approx \lambda(\hat{t})+(t-\hat{t})^{2} \lambda^{\prime \prime}(\hat{t}) / 2$, we have that

$$
f_{H_{1}}(x) \approx \frac{1}{2 \pi i} \int_{c-i \infty}^{c+i \infty} e^{N\left(\lambda(\hat{t})+(t-\hat{t})^{2} \lambda^{\prime \prime}(\hat{t}) / 2\right)} \mathrm{d} t .
$$

Note that this approximation is asymptotically precise (in $N)$. Rearranging, substituting variables $\left(t-\hat{t}=r e^{i \phi}\right)$, and evaluating the Gaussian integral yields

$$
f_{H_{1}}(x) \sim \frac{e^{N \lambda(\hat{t})}}{\sqrt{2 \pi N\left|\lambda^{\prime \prime}(\hat{t})\right|}}
$$

in the usual way for saddlepoint approximations. One can evaluate

$$
\lambda(\hat{t}) \sim 2-2 \ln \frac{N}{x}+\ln \left(\ln \left(\frac{2 N}{x}\right)-1\right)
$$

and $\lambda^{\prime \prime}(\hat{t}) \sim \frac{x^{2}}{2 N^{2}}$. Hence,

$$
f_{H_{1}}(x) \sim \frac{e^{2 N}\left(\ln \left(\frac{2 N}{x}\right)-1\right)^{N}}{\left(\frac{N}{x}\right)^{2 N-1} \sqrt{\pi N}}, \quad N \rightarrow \infty .
$$

By integrating (22) directly, we arrive at

$$
F_{H_{1}}(s) \sim \frac{2^{N}}{(N)^{N} \sqrt{4 \pi N}} \Gamma\left(N+1,2 N\left(\ln \left(\frac{2 N}{s}\right)-1\right)\right) .
$$

The stated asymptotic equivalence for the cumulative distribution then follows from the properties of the upper incomplete gamma function.

Remark 2. Based on (7) and (8), the diversity order of the system without a direct link can be calculated by

$$
d_{1}=N
$$

Remark 3. It is worth highlighting that this asymptotic expression is a refined large deviation result. In fact, to leading order, we have that

$$
F_{H_{1}}(s) \sim \frac{4^{N} e^{-2 N\left(\ln \left(\frac{2 N}{s}\right)-1\right)}}{\sqrt{4 \pi N}}\left(\ln \left(\frac{2 N}{s}\right)-1\right)^{N},
$$


from which we observe that the rate function written for large, finite $N$ is

$$
\begin{aligned}
J_{N}(s) \sim & -\frac{1}{N} \ln F_{H_{1}}(s) \\
\sim & -\ln 4+2\left(\ln \left(\frac{2 N}{s}\right)-1\right) \\
& -\ln \left(\ln \left(\frac{2 N}{s}\right)-1\right)+\frac{\ln 4 \pi N}{2 N} .
\end{aligned}
$$

Applying the large deviation principle directly (instead of the saddlepoint approximation) yields all but the $O\left(\frac{\ln N}{N}\right)$ term for the finite rate function. By using the explicit saddlepoint approximation, however, we obtain additional information about the exponential decay of the distribution as $N$ grows large. Furthermore, we see that, crucially, the asymptotic distribution is accurate for finite $N$ as long as $N \gg s$. In practice, we will be interested in values of s that are inversely proportional to the average received SNR at the destination node. Hence, this condition will be met for large enough SNR. Conversely, when the SNR is on the order of $1 / N$, we can resort to a central limit approximation, or perhaps a refined Edgeworth expansion, for the channel distribution.

\section{B. Perfect Phase Alignment with the Direct Link}

1) Chernoff Bound: We now turn to the case of perfect phase alignment with a direct link. Let $D=\sqrt{\alpha}_{L}\left|h_{L}\right|$. According to the system model, D follows the Rayleigh distribution with the scale parameter $\sigma_{D}=\sqrt{\frac{\alpha_{L}}{2}}$. A Chernoff upper bound of $F_{H_{2}}(s)$ can be written as

$$
F_{H_{2}}(s) \leq \min _{t>0} e^{t s} \mathrm{E}\left[e^{-t D}\right] \prod_{n=1}^{N} \mathrm{E}\left[e^{-t G_{n}}\right],
$$

where $\mathrm{E}\left[e^{-t D}\right]$ can be calculated to be

$$
\mathrm{E}\left[e^{-t D}\right]=1-\frac{\sqrt{\pi \alpha_{L}}}{2} t e^{\frac{\alpha_{L} t^{2}}{4}} \operatorname{erfc}\left(\frac{\sqrt{\alpha_{L}} t}{2}\right) .
$$

Let $z(t)=e^{t s} \mathrm{E}\left[e^{-t D}\right] \prod_{n=1}^{N} \mathrm{E}\left[e^{-t G_{n}}\right]$. Since $z(t)$ can be rewritten as

$$
z(t)=e^{t s} \mathrm{E}\left[e^{-t\left(D+\sum_{n=1}^{N} G_{n}\right)}\right]
$$

we can prove the convexity of $z(t)$ following the same procedure in Proposition 1. Moreover, the optimal value of $t$ for minimizing $z(t)$ can be obtained efficiently by the gradient descent method.

2) Saddlepoint Approximation: The Laplace transform of $f_{D}(s)$ is

$$
L_{D}(t)=1-\frac{\sqrt{\pi \alpha_{L}}}{2} t e^{\frac{\alpha_{L} t^{2}}{4}} \operatorname{erfc}\left(\frac{\sqrt{\alpha_{L}} t}{2}\right) .
$$

Following this, we can write the PDF of $H_{2}$ as

$$
f_{H_{1}}(x)=\frac{1}{2 \pi i} \int_{c-i \infty}^{c+i \infty} L_{D}(t) e^{N \lambda(t)} \mathrm{d} t
$$

where $c$ is greater than the real part of the singularities of the integrand and $\lambda(t)$ is defined in (15). The integral in (31) is dominated for large $N$ by $\hat{t}$, which is the saddlepoint of $\lambda(t)$ given in (18). Invoking the saddlepoint approximation by setting

$$
L_{D}(t) \approx L_{D}(\hat{t}) \quad \text { and } \quad \lambda(t) \approx \lambda(\hat{t})+(t-\hat{t})^{2} \frac{\lambda^{\prime \prime}(\hat{t})}{2}
$$

and evaluating the integral (31), we can arrive at

$$
\begin{aligned}
f_{H_{2}}(x) \sim & L_{D}(\hat{t}) \frac{e^{n \lambda(\hat{t})}}{\sqrt{2 \pi n \lambda^{\prime \prime}(\hat{t})}} \\
\sim & \left(1-\frac{\sqrt{\pi \alpha_{L}} N e^{\frac{\alpha_{L} N^{2}}{x^{2}}} \operatorname{erfc}\left(\frac{\sqrt{\alpha_{L}} N}{x}\right)}{x}\right) \\
& \times \frac{e^{2 N}\left(\ln \left(\frac{2 N}{x}\right)-1\right)^{N}}{\left(\frac{N}{x}\right)^{2 N-1} \sqrt{\pi N}}, \quad N \rightarrow \infty .
\end{aligned}
$$

Based on the asymptotic expression of the complementary error function for large argument [23], we have

$$
\operatorname{erfc}\left(\frac{\sqrt{\alpha_{L}} N}{x}\right) \sim \frac{x e^{-\frac{\alpha_{L} N^{2}}{x^{2}}}\left(1-\frac{x^{2}}{2 \alpha_{L} N^{2}}\right)}{\sqrt{\pi \alpha_{L}} N}, \quad N \rightarrow \infty .
$$

Taking (34) into (33) and integrating (33) directly, we obtain the CDF of $\mathrm{H}_{2}$ :

$F_{H_{2}}(s) \sim \frac{2^{N} \sqrt{N} \Gamma\left(N+1,2(N+1)\left(\ln \left(\frac{2 N}{s}\right)-1\right)\right)}{e^{2} \sqrt{\pi} \alpha_{L}(N+1)^{N+1}}, N \rightarrow \infty$.

According to (7) and (8), the diversity order can then be derived as

$$
d_{2}=N+1 \text {. }
$$

\section{Numerical Results}

In this section, we investigate the outage probability of the IRS-assisted communication systems and evaluate our proposed upper bounds and asymptotic results by Monte Carlo (MC) simulations. We set $\bar{\gamma}=0 \mathrm{~dB}, d_{1}=5 \mathrm{~m}, d_{2}=5 \mathrm{~m}$, $d_{L}=7 \mathrm{~m}, v_{1}=2.5, v_{2}=2.5, v_{L}=3.5$.

Fig. 1 illustrates the outage probability versus the transmit SNR $\gamma_{t}$ when the direct link is blocked. The Chernoff upper bounds and the asymptotic results based on the saddlepoint approximation are compared with MC simulations and the CLT approximation. It can be seen that the CLT approximation brings significant errors when the number of elements is small. This confirms the importance of investigating the tails of the distribution. The Chernoff bounds are more accurate than the CLT method in the high transmit SNR regime. For example, when $N=16$, the Chernoff bound is more accurate for $\gamma_{t}>20 \mathrm{~dB}$. We can also observe that increasing the number of reflecting elements can significantly reduce the transmit SNR to achieve a given outage probability as expected. For example, when the outage probability is $10^{-4}$, the transmit SNR is $30 \mathrm{~dB}$ for $N=8$ and $20 \mathrm{~dB}$ for $N=16$. Since the asymptotic behavior of (25) will appear at very low outage probability for large $N$, the largest $N$ we use in analysis is $N=16$. It is shown that the CLT approximation and the saddlepoint approximation match nicely with the 


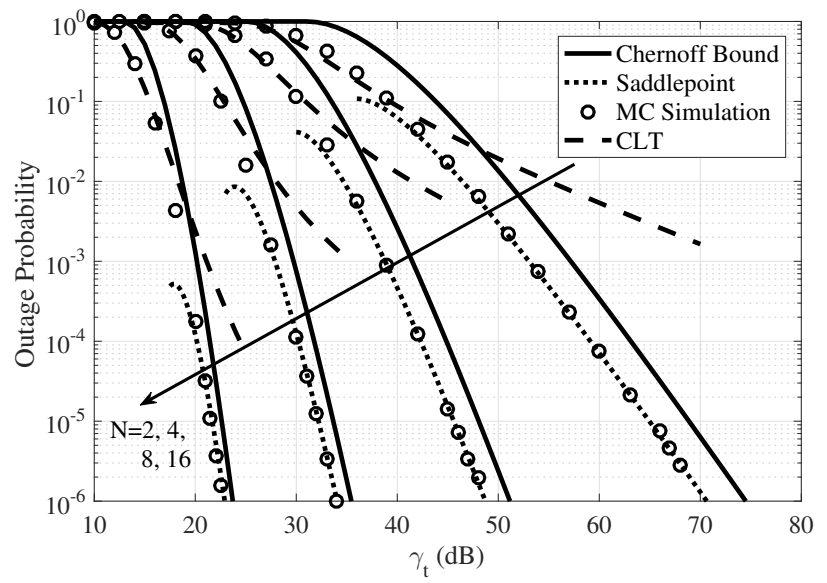

Fig. 1. Outage probability versus transmit SNR without the direct link for different $N$.

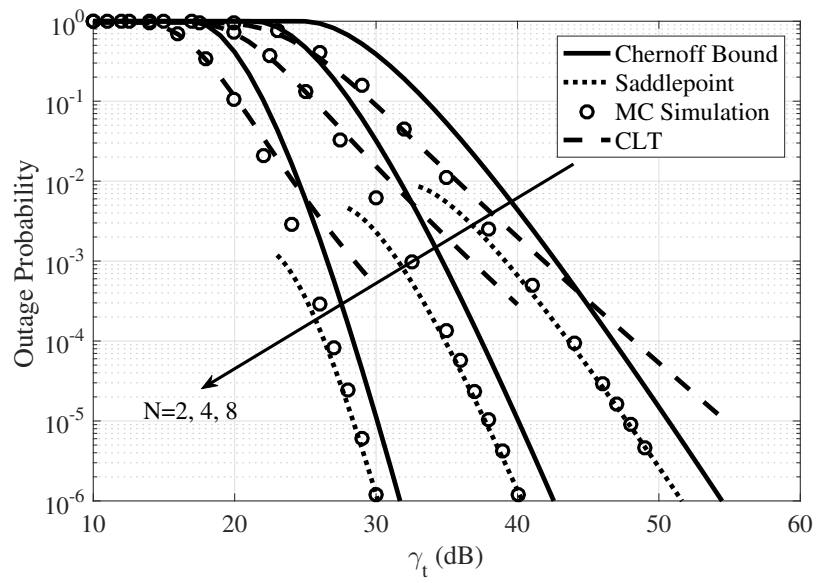

Fig. 2. Outage probability versus transmit SNR with the direct link for different $N$

simulation results in the low and high transmit SNR regimes, respectively. This confirms that the saddlepoint approximation is valid in the tails of the distribution where the CLT method is not accurate.

Fig. 2 shows the outage probability of the system with a direct link. Similar to the case without a direct link, it can be seen that the Chernoff bounds and the asymptotic results based on the saddlepoint approximation are close to the MC results. Comparing Figs. 1 and 2, we can observe a significant performance gain due to the existence of a direct link.

\section{CONCLUSIONS}

In this letter, the outage probability of IRS-assisted communication systems was investigated. It was shown that the Chernoff upper bound is tighter than the CLT approximation for high transmit SNR. Besides, numerical and simulation results confirmed that the reported asymptotic expressions generated by the saddlepoint approximation are accurate in the high transmit SNR regime. We also demonstrated that increasing the number of reflecting elements can significantly reduce the outage probability for the same transmit SNR.

\section{ACKNOWLEDGEMENTS}

This work was supported by EPSRC grant number EP/T02612X/1.

\section{REFERENCES}

[1] C. Liaskos, S. Nie et al., "A new wireless communication paradigm through software-controlled metasurfaces," IEEE Commun. Mag., vol. 56, no. 9, pp. 162-169, 2018.

[2] Q. Wu and R. Zhang, "Intelligent reflecting surface enhanced wireless network: Joint active and passive beamforming design," in Proc. IEEE GLOBECOM, Abu Dhabi, United Arab Emirates, Dec. 2018, pp. 1-6.

[3] Z. Abdullah, G. Chen et al., "A hybrid relay and intelligent reflecting surface network and its ergodic performance analysis," IEEE Wireless Commun. Lett., vol. 9, no. 10, pp. 1653-1657, 2020.

[4] Y. Chen, Y. Wang et al., "Resource allocation for intelligent reflecting surface aided vehicular communications," IEEE Trans. Veh. Technol, pp. 1-1, 2020.

[5] Q. Wu and R. Zhang, "Towards smart and reconfigurable environment: Intelligent reflecting surface aided wireless network," IEEE Commun. Mag., vol. 58, no. 1, pp. 106-112, 2020.

[6] X. Yu, D. Xu et al., "Robust and secure wireless communications via intelligent reflecting surfaces," IEEE J. Select. Areas Commun., to appear.

[7] Y. Han, W. Tang et al., "Large intelligent surface-assisted wireless communication exploiting statistical CSI," IEEE Trans. Veh. Technol, vol. 68 , no. 8, pp. 8238-8242, 2019.

[8] H. Zhang, B. Di et al., "Reconfigurable intelligent surfaces assisted communications with limited phase shifts: How many phase shifts are enough?" IEEE Trans. Veh. Technol, vol. 69, no. 4, pp. 4498-4502, 2020.

[9] D. Li, "Ergodic capacity of intelligent reflecting surface-assisted communication systems with phase errors," IEEE Commun. Lett., 2020.

[10] D. Kudathanthirige, D. Gunasinghe, and G. Amarasuriya, "Performance analysis of intelligent reflective surfaces for wireless communication," in Proc. IEEE ICC, Dublin, Ireland, Jun. 2020, pp. 1-6.

[11] M. Badiu and J. P. Coon, "Communication through a large reflecting surface with phase errors," IEEE Wireless Commun. Lett., vol. 9, no. 2, pp. 184-188, 2020.

[12] T. Wang, G. Chen et al., "Study of intelligent reflective surface assisted communications with one-bit phase adjustments," in Proc. IEEE Global Commun. Conf. (GLOBECOM), Virtual, Dec. 2020, pp. 1-6.

[13] S. Atapattu, R. Fan et al., "Reconfigurable intelligent surface assisted two-way communications: Performance analysis and optimization," IEEE Trans. Wireless Commun., pp. 1-1, 2020.

[14] Z. Cui, K. Guan et al., "SNR coverage probability analysis of RIS-aided communication systems," IEEE Trans. Veh. Technol., vol. 70, no. 4, pp. 3914-3919, 2021.

[15] H. Ibrahim, H. Tabassum, and U. T. Nguyen, "Exact coverage analysis of intelligent reflecting surfaces with Nakagami-m channels," IEEE Trans. Veh. Technol., vol. 70, no. 1, pp. 1072-1076, 2021.

[16] D. Selimis, K. P. Peppas et al., "On the performance analysis of RISempowered communications over Nakagami-m fading," IEEE Commun. Lett., vol. 25, no. 7, pp. 2191-2195, 2021.

[17] J. Gil-Pelaez, "Note on the inversion theorem," Biometrika, vol. 38, no. 3-4, pp. 481-482, 1951.

[18] J. Lyu and R. Zhang, "Hybrid active/passive wireless network aided by intelligent reflecting surface: System modeling and performance analysis," IEEE Trans. Wireless Commun., Early Access.

[19] C. Huang, A. Zappone et al., "Reconfigurable intelligent surfaces for energy efficiency in wireless communication," IEEE Trans. Wireless Commun., vol. 18, no. 8, pp. 4157-4170, 2019.

[20] E. Björnson and L. Sanguinetti, "Rayleigh fading modeling and channel hardening for reconfigurable intelligent surfaces," IEEE Wireless Communications Letters, vol. 10, no. 4, pp. 830-834, 2020.

[21] J. Salo, H. M. El-Sallabi, and P. Vainikainen, "The distribution of the product of independent Rayleigh random variables," IEEE Trans. Antennas Propag., vol. 54, no. 2, pp. 639-643, 2006.

[22] S. Boyd and L. Vandenberghe, Convex Optimization. Cambridge university press, 2004.

[23] F. Olver, Asymptotics and special functions. CRC Press, 1997. 